\title{
Erratum to: Foliations associated to harmonic maps on some complex two ball quotients
}

\author{
Sai-Kee Yeung \\ Department of Mathematics, Purdue University, West Lafayette, IN 47907, USA \\ Email: yeung@math.purdue.edu \\ Erratum to: Science China Mathematics, June 2017 Vol. 60 No. 6: 1137-1148, \\ https://doi.org/10.1007/s11425-016-9044-8 \\ Citation: Yeung S-K. Erratum to: Foliations associated to harmonic maps on some complex two ball quotients. \\ Sci China Math, 2020, 63: 1645, https://doi.org/10.1007/s11425-019-1710-6
}

In [Y, p. 1146, l. 35], it is stated that " $P_{\mathbb{R}} \# T^{2}$ has a two-fold cover that is $T^{2}$ ". This is a mistake. As a result, [Y, Theorem 4.2] should be corrected as follows. The numbering of the references follows $[\mathrm{Y}]$.

Theorem 4.2. Let $M$ be a smooth complex surface of general type with $c_{2}(M)=3$. Then $M$ is an arithmetic complex two ball quotient. Moreover, $M$ is either a fake projective plane or a Cartwright-Steger surface. Hence, there are altogether 101 such surfaces.

Proof. The surface $M$ has to be a complex two ball quotient $B_{\mathbb{C}}^{2} / \Gamma$ as explained in [31, Proposition 2.1], where $\Gamma$ is a cocompact lattice of $P U(2,1)$. The lattice $\Gamma$ is integral as explained in [Y, p. 1146]. An alternate proof is now available through the main result of [EG]. Arithmeticity of $\Gamma$ is a consequence of integrality together with Archimedean rigidity as explained in the first half of [Y, p. 1146], following [Y, Lemma 4.1]. From [21] and [8], it follows from classification of arithmetic lattices with $c_{2}=3$ that $M$ is either a fake projective plane $\left(b_{1}(M)=0\right)$ or a Cartwright-Steger surface $\left(b_{1}(M)=2\right)$. From [BY], it follows that there is exactly one Cartwright-Steger surface, since $M$ can be defined over $\mathbb{Q}$ and the complex conjugate of $M$ is biholomorphic to $M$ (see [BY, Remark 5.1]). Together with the 50 pairs of fake projective planes classified in [21] and [8], this gives rise to 101 smooth surfaces with $c_{2}(M)=3$.

\footnotetext{
References

BY Borisov L, Yeung S-K. Explicit equations of the Cartwright-Steger surface. Épij Géom Algé, 2020, in press

EG Esnault H, Groechenig M. Cohomologically rigid local systems and integrality. Selecta Math (NS), 2018, 24: 42794292

Y Yeung S-K. Foliations associated to harmonic maps on some complex two ball quotients. Sci China Math, 2017, 60: $1137-1148$
}

The online version of the original article can be found at https://doi.org/10.1007/s11425-016-9044-8 